\section{SOI: $\underline{1.1 / \mathrm{TAS}}$ DOI: $\underline{10.15863 / \mathrm{TAS}}$ International Scientific Journal Theoretical \& Applied Science}

\author{
p-ISSN: 2308-4944 (print) e-ISSN: 2409-0085 (online) \\ Year: $2015 \quad$ Issue: 03 Volume: 23 \\ Published: $30.03 .2015 \quad \underline{\text { http://T-Science.org }}$
}

SECTION 26. Radio-technique. Electronics. Telecommunications.
Marina Yur'evna Zvezdina

Doctor of Physical and Mathematical Sciences, associate professor, head of the department of

Don State Technical University, Russia zvezdina_m@mail.ru

Yulia Aleksandrovna Shokova

Candidate of Physical and Mathematical Sciences, associate professor of

Don State Technical University, Russia

Andrey Viktorovich Shokov postgraduate student of

Don State Technical University, Russia

Anton Alekseevich Dymchenko student of

Don State Technical University, Russia

Pavel Alekseevich Parhomenko student of

Don State Technical University, Russia

\title{
ELECTROMAGNETIC SITUATION VISUAL REPRESENTATION NEAR MOBILE BASE STATION ANTENNA
}

\author{
Abstract: The results of electromagnetic situation research near GSM and TETRA mobile base station antenna \\ are given. The maximum size of sanitary protection zone is estimated and vertical energy flux density distribution is \\ represented visually. It is shown that it is safe to place low-back-lobe aerial on the rooftop regardless the \\ transmitter power. Physical interpretation of the results is given. \\ Key words: electromagnetic situation, energy flux density, base station antenna, visual representation of field \\ structure. \\ Language: Russian \\ Citation: Zvezdina MY, Shokova YA, Shokov AV, Dymchenko AA, Parhomenko PA (2015) \\ ELECTROMAGNETIC SITUATION VISUAL REPRESENTATION NEAR MOBILE BASE STATION \\ ANTENNA. ISJ Theoretical \& Applied Science 03 (23): 10-17.

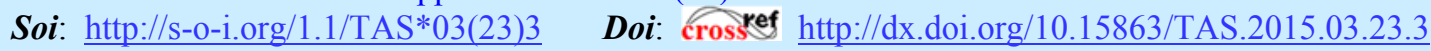

\section{ВИЗУАЛИЗАЦИЯ ЭЛЕКТРОМАГНИТНОЙ ОБСТАНОВКИ ВБЛИЗИ АНТЕННЫ БАЗОВОЙ СТАНЦИИ СИСТЕМЫ ПОДВИЖНОЙ СВЯЗИ}

Аннотация: Приводятся результаты исследований электромагнитной обстановки вблизи мест размещения антенн базовых станциий стандартов GSM и TETRA. Выполнена оценка максимальных размеров санитарно-защитной зоны и визуализачия распределения плотности потока энергии в вертикальной плоскости. Показано, что для антенн с низким уровнем заднего излучения нахождение на крыше в месте установки антенны является безопасным независимо от мощности передатчика. Дается физическая трактовка результатов.

Ключевые слова: электромагнитная обстановка, плотность потока энергии, антенны базовых станциий, визуализация структуры поля.

В современных условиях системы подвижной связи нашли широкое применение во всех областях человеческой деятельности. Так, системы сотовой связи наряду с традиционной передачей речевых сообщений предоставляет и такие услуги, как мобильный Интернет, передача видео сообщений, Интернет-телевидение [1]. Для организации ведомственных и корпоративных сетей применяются системы транкинговой связи, т.е. радиально-зоновые системы подвижной УКВсвязи [2]. В обоих случаях для оказания услуг в полной мере антенны базовых станций размещают вблизи потребителей услуг, в том числе и на крышах жилых зданий [3; 4, с.80]. Размещение антенн базовых станций в местах с наибольшей плотностью населения приводит к тому, что они становятся источниками ухудшения экологической ситуации по электромагнитному фактору, поскольку антенны устанавливаются на небольшой высоте от поверхности земли (или крыши здания), а 
максимальное направление излучения ориентировано в сторону земли.

Для оценки электромагнитной обстановки вблизи них Государственной санитарноэпидемиологической службой Российской Федерации утверждены гигиенические требования к размещению и эксплуатации передающих радиотехнических объектов [5], а также Методические указания [6] по определению плотности потока энергии (ППЭ) электромагнитного поля в местах размещения радиосредств, работающих в диапазоне частот 300 МГц - 300 ГГц. Перечисленные выше документы определяют в указанном частотном диапазоне предельно допустимый уровень

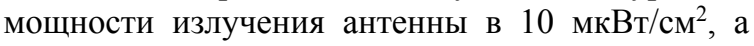
также методику измерения плотности потока энергии, создаваемого радиотехническим средством. На этапе проектирования для определения мест размещения антенн базовых станций целесообразно использовать методы расчетного прогнозирования. Данные методы подразумевают наряду с использованием соотношений, описанных в $[6 ; 7$, с.146], привлечение для их программной реализации основных положений электродинамики. Примером программы, построенной на использовании перечисленных выше методик и предназначенной для расчета размеров санитарно-защитных зон в горизонтальной плоскости, является платная программа SANZONE [8]. В то же время на практике имеется потребность в знании структуры электромагнитного поля антенны и в вертикальной плоскости. Для решения указанной задачи авторами в языковой среде MathCad 15 разработан пакет из двух программ, позволяющий визуализировать структуру электромагнитного поля как в горизонтальной плоскости на крыше здания, так и в вертикальной плоскости в заданном направлении [9, с.70]. С использованием разработанного пакета программ в работе [10] авторами были выполнены исследования, направленные на визуализацию структуры электромагнитного поля для ряда антенн систем сотовой связи. Однако вопросы оценки размеров санитарно-защитной зоны в указанной работе не рассматривались.

Цель данного доклада - исследование структуры электромагнитного поля наиболее часто применяемых антенн базовых станций систем подвижной связи.

Для проведения исследований по оценке экологической обстановки вблизи антенны базовой станции системы подвижной связи необходимо сначала определить стандарт связи, а также частотный диапазон, в котором они функционируют. Для систем связи в настоящее время наблюдается постоянное совершенствование технологий беспроводного доступа, обусловленное необходимостью эффективного использования ограниченного радиочастотного спектра и увеличением скорости передачи данных $[11$, с.50]. В связи с этим на мировом рынке сотовой связи представлены как более старые стандарты IMT-2000, так и его более новые разновидности - $3 G$ и $4 G$, имеющие меньшую потребность в частотном ресурсе и функционирующие в дециметровом диапазоне частот. Следует отметить, что в соответствии с рекомендациями Международного союза электросвязи МСЭ- $R$ M.1036-3 [12, с.1] для подвижной радиосвязи стандарта IMT-2000 определены частоты 806-960 МГц, 1710-1885 МГц и 2500-2690 МГц. Среди стандартов транкинговых сетей связи, как следует из $[2,13]$, широкой поддержкой большинства крупных мировых производителей оборудования и администрацией связи различных стран пользуется стандарт TETRA. Для него в Европе закреплены диапазоны: за службами безопасности 380-395 МГц, для коммерческих организаций - 410-470 МГц.

В табл. 1 по данным из [14, с.43; 15] приведены основные электрические параметры антенн базовых станций ряда систем подвижной связи. При проведении исследований влияния электрических параметров антенн базовых станций на распределение плотности потока энергии вблизи места ее установки будем использовать модель для радиотехнических средств УКВ и ОВЧ диапазонов, описанную в [7, c. 146]. Данная модель предполагает, что излучающая антенна размещается на крыше здания высотой $h$. Здание представляет собой прямоугольный параллелепипед, координаты $A$, $B, C, D$ углов крыши которого известны. Положение антенны на крыше здания фиксировано и определяется координатами $F=\left\{x_{f}, y_{f}, z_{f}\right\}$. На высоту размещения антенны накладывается ограничение $z_{f}>2 L^{2} / \lambda$, где $L-$ наибольший размер по вертикали источника излучения [7, с.53]; $\lambda=c / f$ - рабочая длина волны; $c=3 \cdot 10^{8}$ м/с - скорость света; $f$-рабочая частота, в Гц.

При введенных ограничениях на высоту подвеса антенны ее характеристика направленности в главных плоскостях $F(\theta)$, $F\left(\varphi^{\prime}\right)$ может задаваться на основе паспортных данных $\left(\theta, \varphi^{\prime}\right.$ - угол места и азимут в системе координат, связанной с фазовым центром антенны и направлением максимального излучения антенны). Требуется определить максимальный размер санитарно-защитной зоны 
(C33), a также построить распределение плотности потока энергии от антенны в горизонтальной плоскости над крышей и в вертикальной плоскости, проходящей через фазовый центр антенны.

\section{Электрические параметры типовых антенн базовых станций.}

Таблица 1

\begin{tabular}{|c|c|c|c|c|c|}
\hline \multirow{2}{*}{ Модель антенны } & \multirow{2}{*}{$\begin{array}{c}\text { Частотный } \\
\text { диапазон, } \\
\text { МГц } \\
\end{array}$} & \multirow{2}{*}{$\begin{array}{c}\text { Коэффициент } \\
\text { усиления } \\
\text { антенны, дБ } \\
\end{array}$} & \multicolumn{2}{|c|}{ Ширина главного лепестка, град } & \multirow{2}{*}{$\begin{array}{c}\text { Максимально } \\
\text { допустимая входная } \\
\text { мощность, Вт }\end{array}$} \\
\hline & & & в гор. пл. & в вертик. пл. & \\
\hline \multicolumn{6}{|c|}{ Антенны транкинговой связи стандарта TETRA } \\
\hline RAV-2UL-90 & $400-430$ & 8 & 37 & 90 & 400 \\
\hline RAV-4UL-90 & $400-430$ & 11 & 18 & 90 & 400 \\
\hline RAO-4U-120 & $400-470$ & 8 & 36 & 120 & 400 \\
\hline RAX-2UL-70 & $400-430$ & 9 & 36 & 70 & 400 \\
\hline RAX-4UL-70 & $400-430$ & 12 & 18 & 70 & 400 \\
\hline RAO-2U-60 & $380-440$ & 10 & 36 & 60 & 500 \\
\hline \multicolumn{6}{|c|}{ Антенны системы сотовой связи стандарта $G S M(3 G)$} \\
\hline RAO-11GL-60 & $860-970$ & 11 & 30 & 60 & 50 \\
\hline RAO3-10GH-60 & $1710-1880$ & 13 & 18 & 60 & 50 \\
\hline RAO-14GL-70 & $860-970$ & 14 & 15 & 70 & 50 \\
\hline \multicolumn{6}{|c|}{ Антенны системы сотовой связи стандарта $L T E(4 G)$} \\
\hline RAX-14Yota-70 & $2400-2700$ & 14.2 & 8 & 65 & 50 \\
\hline
\end{tabular}

Решение начнем с первой сформулированной задачи, которое будем выполнять без учета влияния здания, на котором размещается антенна. В соответствии с ней величина ППЭ $\left[\right.$ мкВт/ $\left./ \mathrm{cm}^{2}\right]$, создаваемого антенной, рассчитывается по найденному значению амплитуды напряженности электрического поля $E[\mathrm{~B} / \mathrm{M}]$ выражением:

$$
\begin{gathered}
\Pi=E^{2} / 3.77, \\
E=\sqrt{30 P G \eta} k_{\phi} F(\alpha) F(\varphi) / R,
\end{gathered}
$$

где $P$ - мощность на входе антенно-фидерного тракта, Вт; $G$ - коэффициент усиления антенны относительно изотропного излучателя в направлении максимального излучения; $\eta$ коэффициент полезного действия в антеннофидерном тракте; $k_{\phi}=1,15 . .1,3$ - коэффициент, учитывающий двухлучевую модель распространения в условиях статистически неоднородной среды; $R$ - расстояние от антенны до расчетной точки (наклонная дальность); $\alpha, \varphi$ - угловые координаты точки наблюдения.

При оценке электромагнитной обстановки в местах размещения радиотехнических средств будем ориентироваться на наихудший с точки зрения вреда по экологическому фактору вариант параметров антенны. C учетом данного предположения будем рассчитывать максимально возможный размер санитарно-защитной зоны в направлении максимального излучения антенны. Поскольку в данном направлении $F(\theta)=F\left(\varphi^{\prime}\right)=1$, то максимальный размер в указанном направлении может быть найден с использованием соотношения:

$$
R_{\max }=\sqrt{\frac{30 P G \eta}{3.77 a}},
$$

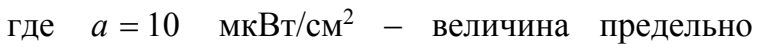
допустимого уровня плотности потока энергии в рассматриваемом диапазоне частот [5], $k_{\phi}=1$.

На рис.1 приведены рассчитанные значения $R_{\max }$ для антенн базовых станций из табл.1. В качестве мощности на входе антенно-фидерного тракта бралась максимально допустимая мощность антенны.

Анализ полученных результатов показывает, что максимальное расстояние до близстоящего жилого здания не должно быть меньше 22 м (антенна RAO-11GL-60) для систем стандарта $3 G$ и от 49 м (антенна RAV-2UL-90) до 69 м (антенна RAX-4UL-70) при размещении антенн стандарта TETRA. Приведенные расстояния справедливы на уровне размещения фазового центра антенны и в направлении ее максимального излучения в 
предположении, что мощность сигнала на входе антенны равна максимально допустимой мощности сигнала. В реальных условиях мощность антенн базовых станций стандарта TETRA намного меньше заявленной в табл.1. Так, в [13] говорится о том, что мощность антенны базовой станции составляет не 400 Вт, а только
20 Вт. Таким образом, максимальный размер С33 может составлять не 69 м, а всего 16 м.

Рассмотрим влияние направленных свойств антенны на распределение создаваемой ею плотности потока энергии для случая размещения антенны на крыше здания.

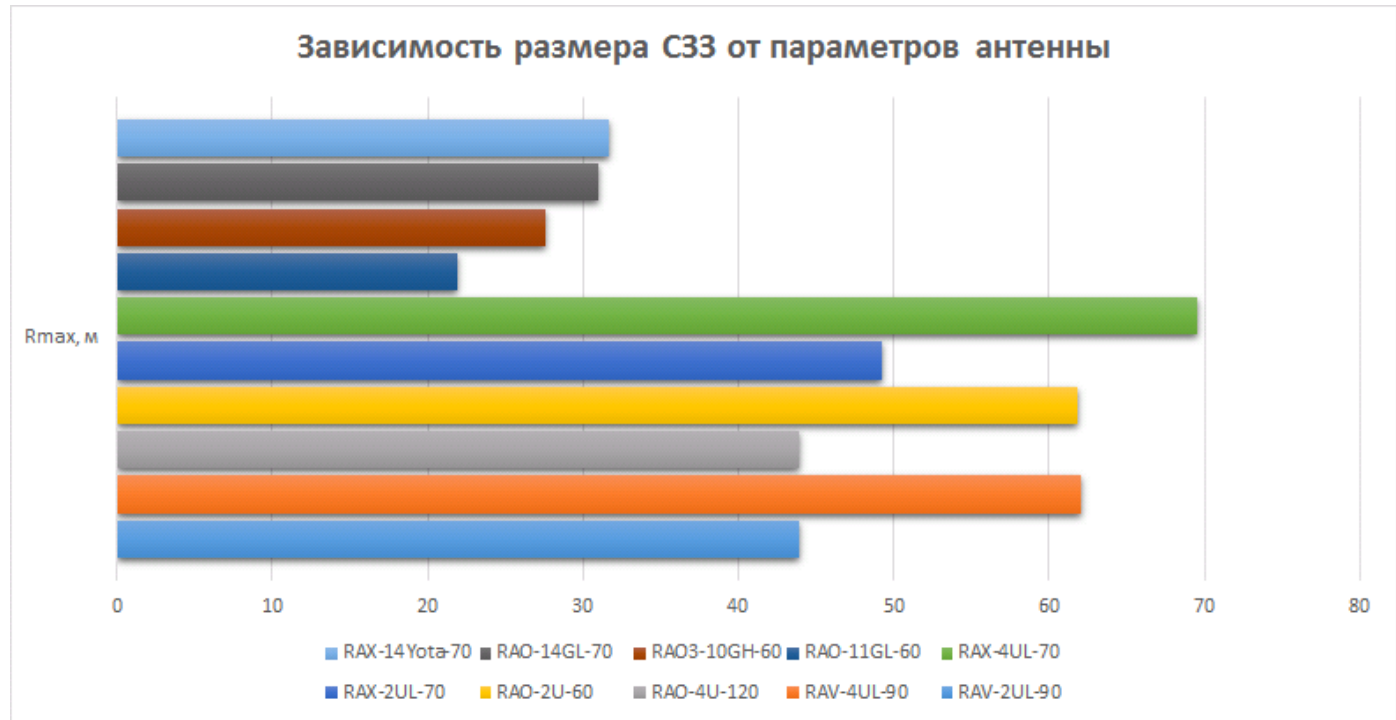

Рисунок 1 - Максимальные размеры санитарно-защитных зон для исследуемых антенн базовых станций.

Как и в предыдущем вопросе, для нахождения плотности потока энергии будем использовать соотношения (1) и (2). При вычислении структуры поля в плоскости особую сложность представляет сопряжение систем координат, связанных с фазовым центром антенны, с центром здания, а также необходимость учета различия между углами $\theta$ и $\alpha, \varphi^{\prime}$ и $\varphi$. Разработанные авторами на основе методики из [7, с.146] программы, описанные в [9, с.70], позволяют учесть все перечисленные выше особенности. В связи с этим будем далее их использовать.

Рассмотрим структуру плотности потока энергии в двух плоскостях: горизонтальной, расположенной на высоте 2 м над поверхностью параллельной земле плоской крыши здания и ограниченной ее размерами, и вертикальной, проходящей через фазовый центр антенны и направление максимального излучения. Предположим, что углы крыши располагаются, как в [10], в точках с координатами $A(0,0,30)$, $B(-7,4,30), C(-13.5,-4,30), D(-2.5,-8.5,30)$.

В качестве исследуемых рассмотрим четыре варианта устанавливаемой на крыше антенны антенну транкинговой связи RAO-2U-60 и три антенны сотовой связи: RAO3-10GH-60, RAO14GL-70 и RAX-14Yota-70. Данные варианты выбирались из условия либо максимальной мощности излучения (первый случай), либо максимального значения коэффициента усиления (остальные случаи). Поскольку справедливость выбранной модели определяется высотой размещения антенны и ее геометрическими размерами, то в табл.2 приводятся параметры выбранных антенн и требуемая минимальная высота ее размещения. С учетом данных табл.2 в языковой среде MathCad 15 были проведены исследования структуры электромагнитного поля перечисленных антенн. При проведении исследований полагалось, для рассмотрения контрастных вариантов размещения антенны последняя размещалась посередине одной из кромок, a направление ее максимального излучения ориентировалось в сторону от здания, перпендикулярно краю крыши. Такое размещение относится, как показано в [3], к одному из типичных вариантов. Результаты исследований приведены на рис.2-5.

Выполним анализ полученных результатов. Начнем со сравнения антенн с направленными и ненаправленными свойствами. На рис.2 приводятся результаты исследований распределения ППЭ, создаваемой в горизонтальной плоскости (рис.2,a), проходящей на высоте 2 м над поверхностью крыши, а также в вертикальной плоскости (рис.2,б) для антенны, 
чья диаграмма направленности совпадает с диаграммой направленности элементарного электрического вибратора (ненаправленная диаграмма направленности):

$$
F(\theta)=|\sin (\theta)|, \quad F\left(\varphi^{\prime}\right)=1,
$$

мощность излучения составляет $50 \mathrm{BT}$, а рабочая частота равна 915 МГц.

Для сравнения на рис.3 приводится распределение плотности потока энергии, создаваемой в вертикальной плоскости антенны RAO-14GL-70, являющейся аналогом антенны с рис.2, но обладающей направленными свойствами. Паспортные данные для нее взяты на перечня оборудования для базовых станций на сайте [15]. Сечение горизонтальной плоскостью не приводится, поскольку наблюдаемый уровень ППЭ на несколько порядков меньше предельно допустимого уровня.

Таблица 2

Электрические параметры исследуемых антенн базовых станций.

\begin{tabular}{|c|c|c|c|c|c|c|c|}
\hline $\begin{array}{c}\text { Модель } \\
\text { антенны }\end{array}$ & $\begin{array}{c}\text { Рабочая } \\
\text { частота } f, \\
\text { МГц }\end{array}$ & \begin{tabular}{|} 
Коэффициент \\
усиления \\
антенны $G$, \\
дБ
\end{tabular} & \begin{tabular}{|c} 
Максимально \\
допустимая \\
входная \\
мощность $P$, \\
Вт
\end{tabular} & $\begin{array}{c}\text { Длина } \\
\text { волны } \\
\lambda, \mathrm{m}\end{array}$ & $\begin{array}{c}\text { Максимальный } \\
\text { размер } \\
\text { антенны по } \\
\text { вертикали } L, \text { м }\end{array}$ & \begin{tabular}{|c} 
Высота \\
размещения \\
фазового \\
центра \\
антенны $z_{f}$, \\
м \\
\end{tabular} & 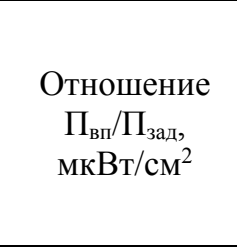 \\
\hline $\begin{array}{c}\text { RAO-2U- } \\
60 \\
\end{array}$ & 415 & 10 & 500 & 0.723 & 1.235 & 4.2 & $187.9 / 7.8 \cdot 10^{-2}$ \\
\hline $\begin{array}{c}\text { RAO3- } \\
10 \mathrm{GH}-60 \\
\end{array}$ & 1795 & 13 & 50 & 0.167 & 0.52 & 3.2 & $14.2 / 8 \cdot 10^{-4}$ \\
\hline $\begin{array}{c}\text { RAO- } \\
14 \mathrm{GL}-70\end{array}$ & 915 & 14 & 50 & 0.328 & 1.018 & 6.3 & $105.8 / 2.1 \cdot 10^{-2}$ \\
\hline $\begin{array}{c}\text { RAX- } \\
14 \text { Yota-70 }\end{array}$ & 2550 & 14.2 & 50 & 0.118 & 0.45 & 3.5 & $14.6 / 5.9 \cdot 10^{-3}$ \\
\hline
\end{tabular}

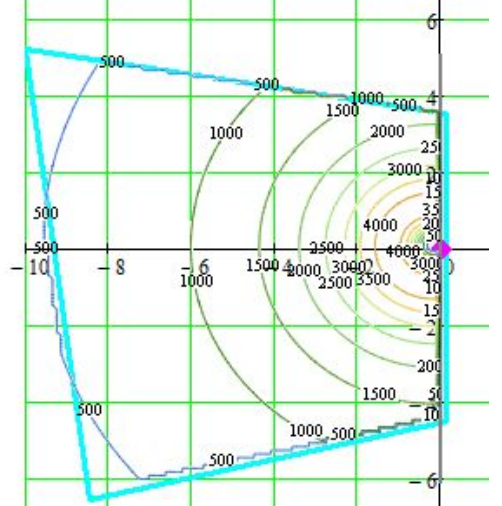

a)

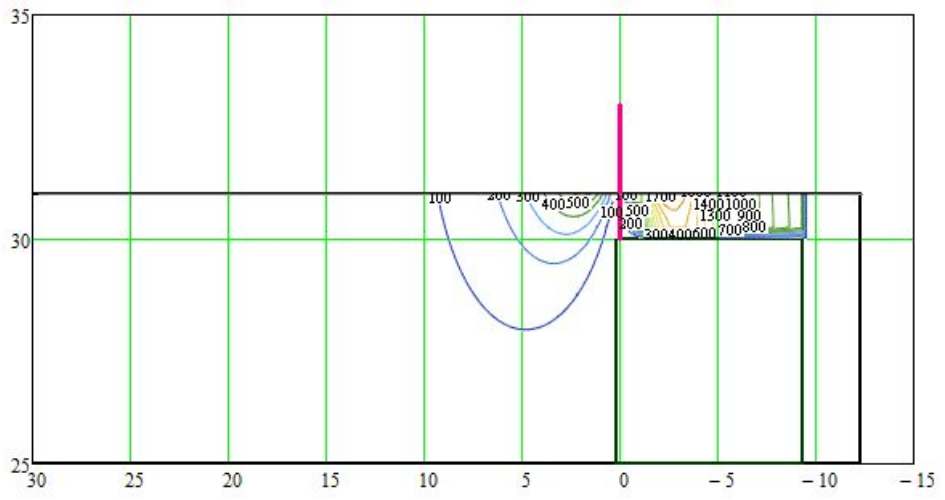

б)

Рисунок 2 - Распределение ППЭ вблизи элементарного электрического вибратора.

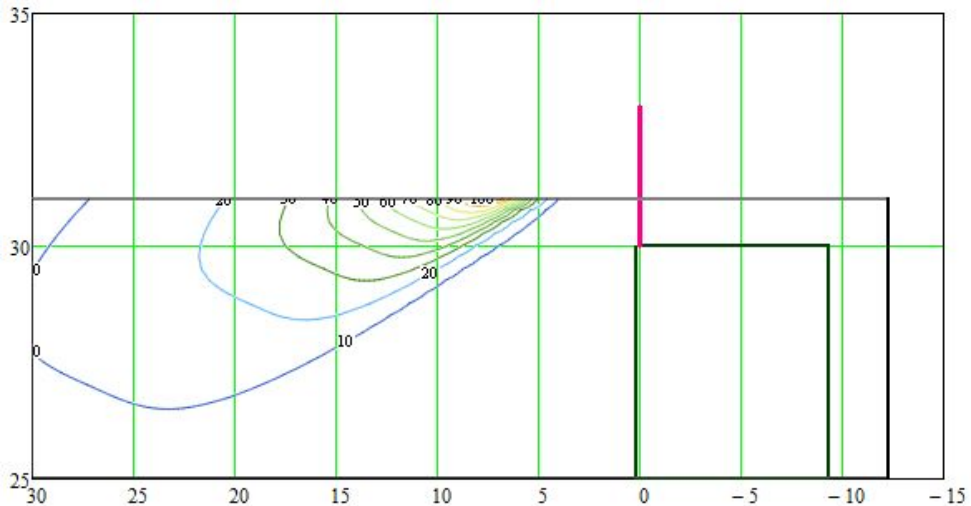

Рисунок 3 - Распределение ППЭ в вертикальной плоскости для антенны RAO-14GL-70. 


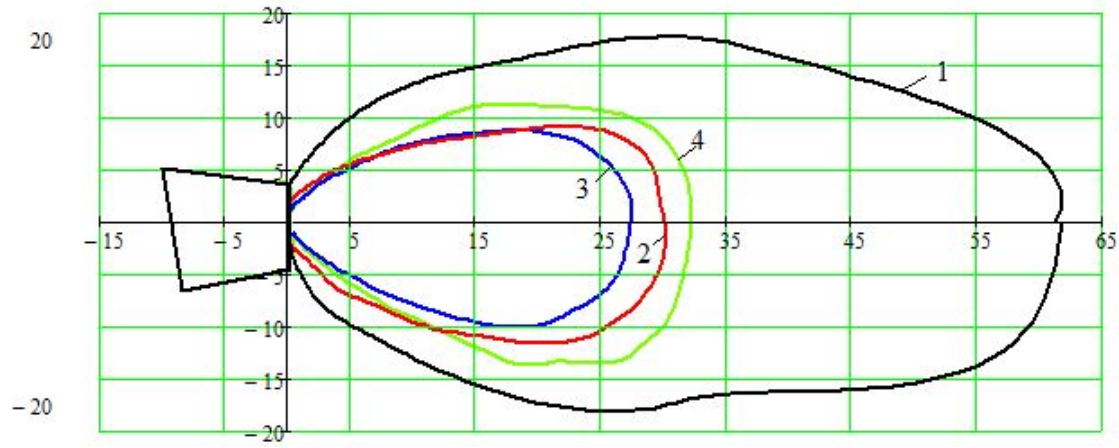

Рисунок 4 - Размеры санитарно-защитных зон в горизонтальной плоскости.

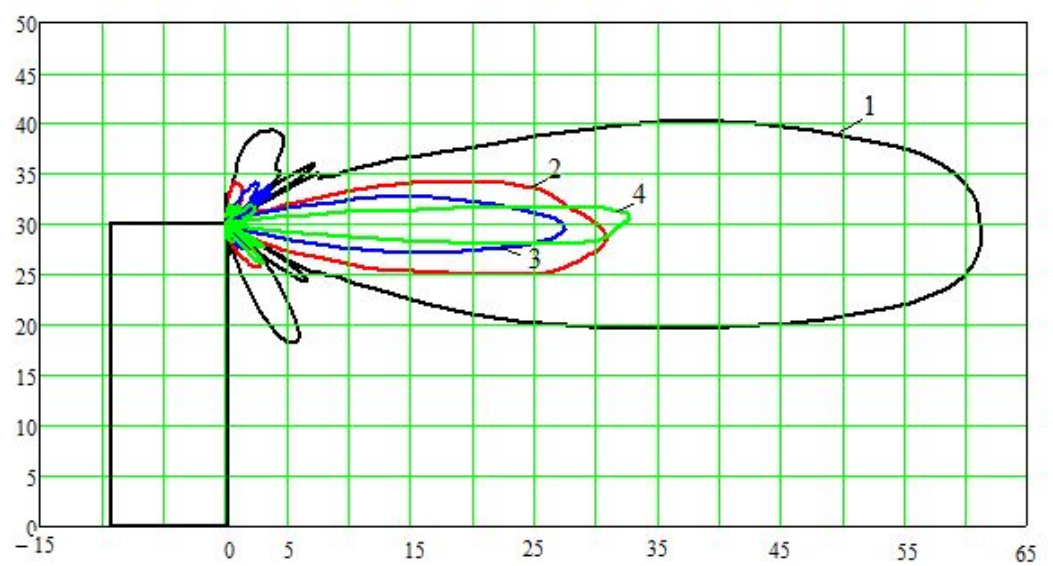

Рисунок 5 - Размеры санитарно-защитных зон в вертикальной плоскости.

Выполненный анализ влияния направленных свойств антенны на распределение ППЭ позволил сделать следующие выводы:

- антенны с диаграммой направленности элементарного электрического вибратора на крыше здания на высоте 2 м от поверхности крыши создают уровень плотности потока

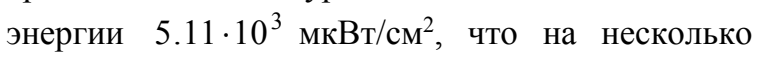
порядков превышает предельно допустимое значение;

- антенны, у которых диаграммы направленности имеют уровень заднего излучения не более -20 дБ, обеспечивают уровень ППЭ на крыше не выше 0.021 мкВт/см².

Полученные результаты имеют четкую физическую трактовку. В горизонтальной плоскости, поскольку диаграмма направленности элементарного электрического вибратора является изотропной, просматриваются дуги концентрических окружностей, центр которых совпадает с центром размещения антенны. В вертикальной плоскости над крышей за счет отражения излучаемого антенной электромагнитного поля наблюдается увеличение уровня плотности потока энергии [16, с.415]. В переднем полупространстве отражающая поверхность отсутствует, что приводит к тому, что здесь просматриваются линии одинакового уровня, по форме совпадающие с сечением вертикальной плоскостью диаграммы направленности элементарного электрического вибратора. Графическое отличие от окружности на рис. 2,б связано с неравномерностью масштаба по координатным осям.

Выявленные закономерности подтверждаются границами санитарно-защитных зон, сечения для которых горизонтальной и вертикальной плоскостью показаны на рис. 4 и 5 соответственно. На данных рисунках использованы следующие обозначения: 1 границы антенны RAO-2U-60; 2 - антенны RAO14GL-70; 3 - антенны RAO3-10GH-60; 4 антенны RAX-14Yota-70, т.е. по мере увеличения рабочей частоты. При изучении данного вопроса полагалось, что горизонтальное сечение проходит через направление максимального излучения антенны, а вертикальное - через антенный излучатель. Влияние краевых эффектов от крыши на рис. 4 и 5 не учитывалось.

Анализ остальных исследуемых антенн показал, что антенны с низким уровнем заднего излучения создают на крыше, как следует из данных табл. 2, уровень плотности потока энергии, намного меньший предельно допустимого независимо от величины максимально допустимой входной мощности. Величина входной мощности антенны оказывает существенное влияние на максимальный размер 
С33. Направленные свойства антенны влияют на форму C33: границы области практически повторяют форму диаграммы направленности антенны.

Полученные результаты, полученные на основе созданного авторами программного обеспечения, позволяют обосновывать рекомендации по размещению антенн базовых станций подвижной связи на крышах жилых зданий. В частности, можно рекомендовать для обеспечения безопасного уровня ППЭ в пределах крыши жилого здания размещать антенны подвижной связи по периметру крыши, чтобы направления их максимального излучения были ориентированы во внешнюю область, а не на небольшом расстоянии вокруг единого фазового центра. Уменьшение размеров санитарнозащитной зоны достигается снижением мощности передатчика. Так, уменьшение мощности передатчика в $N$ раз сокращает максимальный размер санитарно-защитной зоны в $\sqrt{N}$ раз.

\section{References:}

1. (2015) Megafon, http://rostov.megafon.ru/ 7.03.2015)

2. (2015) Trankingovaya radiosvyaz', trank, tsifrovye sistemy DMR, NEXEDGE, Available: http://www.radio-center.ru/trank.shtml (Accessed: 9.03.2015).

3. (2011) O bazovykh stantsiyakh GSM, 3G i LTE - dostupno i prosto, Available: http://tele2life.ru/1/content/view/69/66/

(Accessed: 7.03.2015).

4. Gurevich ES, Popova TS, Moiseenko OA (2014) Aktual'nye zadachi sanitarnoepidemiologicheskikh ekspertiz razmeshcheniya bazovykh stantsiy besprovodnoy radiotelefonnoy svyazi Zdorov'e. Meditsinskaya ekologiya. Nauka. 2014. Volume 58. № 4. pp.80-83, Available: https://yadi.sk/i/vX4p-x7mZKuuU (Accessed: 9.03.2015).

5. (2003) SanPin 2.1.8/2.4.1383-03. Gigienicheskie trebovaniya k razmeshcheniyu i ekspluatatsii peredayushchikh radiotekhnicheskikh ob"ektov. Utv. Glavnym gos. sanitarnym vrachom Rossii June, 9, 2003. Registration number 4710. Data vvedeniya June $30,2003$.

6. (2003) MUK 4.3-1167-02. Opredelenie plotnosti potoka energii elektromagnitnogo polya $\mathrm{v}$ mestakh razmeshcheniya radiosredstv, rabotayushchikh v diapazonakh chastot 300 MGts - 300 GGts. Utv. Predsedatelem Gos. sanitarno-epidemiologicheskogo nadzora RF 7.10.2002Г., Available: http://rfcmd.ru/sphider/docs/project/MUK\%204 3.1167-02.htm (Accessed: 7.03.2015)

7. Spodobaev YM, Kubanov VP (2000). Osnovy elektromagnitnoy ekologii. Moskow: Radio i svyaz', $240 \mathrm{p}$.
8. (2013) Program SANZONE, Available: http://www.ing-tv.ru/index/sanzone/0-53

(Accessed: 7.03.2015).

9. Zvezdina MY, et all (2015) Programmnyy kompleks dlya vizualizatsii elektromagnitnoy obstanovki vblizi antenny sotovoy svyazi. Sbornik nauchn. trudov po materialam Mezhdunarodnoy nauchno-prakticheskoy konferentsii «Teoreticheskie i prakticheskie voprosy nauki i obrazovaniya». Tambov, January, 31, 2015г. Part 10. Tambov: OOO "Konsaltingovaya kompaniya Yukom ", 2015. 164 p./ pp.70-74.

10. Zvezdina MY, Shokova YA, Shokov AV (2015). Vliyanie parametrov antenn trankingovoy i sotovoy svyazi na elektromagnitnuyu obstanovku vblizi mest ustanovki, Universum: Tekhnicheskie nauki: elektronnyi nauchnyi zhurnal. 2015. № 2 (15), Available:

http://7universum.com/ru/tech/archive/item/196 1 (Accessed: 8.03.2015).

11. Skrynnikov V (2014) 4G: sostoyanie rynka, dinamichnoe razvitie tekhnologiy (LTEAdvanced), Tekhnologiya i sredstva svyazi, №4, pp.50-55.

12. (2007) Rekomendatsiya ITU-R M.1036-3. Plany razmeshcheniya chastot dlya vnedreniya nazemnogo segmenta sistemy Mezhdunarodnoy podvizhnoy elektrosvyazi-2000 (IMT-2000) v polosakh 806-960 MGts, 1710-2025 MGts, 2110-2200 MGts i 2500-2690 MGts. (Vopros ITU-R 229/8), Available: https://www.itu.int/dms pubrec/itu-r/rec/m/RREC-M.1036-3-200707-S!!PDF-R.pdf (Accessed: 9.03.2015).

13. Trankingovye sistemy svyazi Motorola Dimetra standarta TETRA, Available: http:/www.elcomplus.ru/production/pmr348.html (Accessed: 9.03.2015). 
14. Voskresenskiy DI, et al. (2008) Antennye sistemy bazovoy stantsii sistemy sotovoy svyazi standarta GSM i 3G (obzor), Antenny. № 6 (133). pp.43-52.

15. (2015) Bazovoe antenno-fidernoe oborudovanie. Sayt «Radial», Available: http://www.radial.ru/catalog/antennas/vertical/a 3 cdma/ (Accessed: 9.03.2015).

16. Pimenov YV (2008) Lineynaya makroskopicheskaya elektrodinamika. Vvodnyy kurs dlya radiofizikov i inzhenerov, Dolgoprudnyy: Intellekt, $536 \mathrm{p}$. 